\title{
Learning Target Dynamics While Tracking Using Gaussian Processes
}

Clas Veibäck, Jonatan Olofsson, Tom Rune Lauknes and Gustaf Hendeby

The self-archived postprint version of this journal article is available at Linköping University Institutional Repository (DiVA):

http://urn.kb.se/resolve?urn=urn:nbn:se:liu:diva-168871

N.B.: When citing this work, cite the original publication.

Veibäck, C., Olofsson, J., Lauknes, T. R., Hendeby, G., (2020), Learning Target Dynamics While

Tracking Using Gaussian Processes, IEEE Transactions on Aerospace and Electronic Systems, 56(4), 2591-2602. https://doi.org/10.1109/TAES.2019.2948699

Original publication available at:

https://doi.org/10.1109/TAES.2019.2948699

Copyright: Institute of Electrical and Electronics Engineers http://www.ieee.org/index.html 


\title{
Learning Target Dynamics While Tracking Using Gaussian Processes
}

\author{
Clas Veibäck, Member, IEEE, Jonatan Olofsson, Tom Rune Lauknes, Member, IEEE, and Gustaf Hendeby, Senior \\ Member, IEEE
}

\begin{abstract}
Tracked targets often exhibit common behaviours due to influences from the surrounding environment, such as wind or obstacles, which usually are modelled as noise. Here these influences are modelled using sparse Gaussian processes that are learned online together with the state inference using an extended Kalman filter. The method can also be applied to time-varying influences and identify simple dynamic systems. The method is evaluated with promising results in a simulation and a real-world application.
\end{abstract}

Index Terms-Sparse Gaussian Process, Target Tracking, Extended Kalman Filter, Online Learning, Identification

\section{INTRODUCTION}

In target tracking it is common to use general purpose motion models, e.g. the near constant velocity $(\mathrm{NCV})$ model, to predict the behaviour of targets. However, particular behaviours of the targets are often observed and it is desirable to use this information to improve predictions over time. The behaviour of all targets may be influenced by a common process, e.g., in the form of

- preferred paths in open terrain or indoors;

- obstacles that are avoided or common destinations;

- velocity or acceleration profiles in racing events;

- wind affecting flying vehicles; and

- currents in the water affecting animals and ships.

For a single dynamical system an approximate model can often be determined beforehand, but it might be difficult to obtain an accurate model due to unknown influences from the environment or variations over time. For such systems estimation and prediction could be improved by using the available information to learn and gradually improve and adapt the model.

In target tracking it has long been standard practice to use interacting multiple models (IMM) [1] to consider various behaviours for targets. However, the behaviours are determined at design time and are not adapted to new data, so this approach is not suitable for the influences mentioned previously.

An approach to model a behaviour is described in [2], where the system pulls towards a given mean. The mean can be based on historic data and therefore work well to model preferred paths and velocity profiles. The method is shown in [3] and [4] to improve predictions of ship trajectories. Similar ship prediction problems are solved using a nearest neighbour approach in [5] and oblong process noise along routes in [6]. However, the methods grow in complexity with the size of the datasets, since new data is matched against all historic data, and it is not obvious from the methods how to appropriately compute the mean. Other approaches to model particular behaviour are described in [7] and [8], where road maps are used to position cars. These approaches are, however, limited to applications where the map is known in advance.

In computer vision, it is common to model motion patterns across the image. These motion patterns show some resemblance to the method proposed in this paper. In [9], [10] and [11] trajectories in images are extracted and clustered, whereupon the estimated positions and velocities are used to train a Gaussian process motion model for each cluster. These are offline methods, where the motion pattern is only loosely coupled with the target motion model. An online adaptation is proposed in [9] by recomputing the model using only the latest data. Another approach is taken in [12] where the shapes of clustered trajectories instead are modelled as sparse Gaussian processes.

This paper proposes an approach for joint state estimation and online learning of influences in a dynamical system using recursive Gaussian process regression. The system function consists of a known linear term and an unknown nonlinear term of influence functions. The influence functions are modelled as independent Gaussian processes (GP) [13] with statedependent input. Either they model the entire dynamics, in which case the linear term reduces to a prior mean function of the Gaussian processes, or they model a particular influence on the system that is difficult to determine at design time. The advantage of the latter is that the influence can have a lower dimension than the state vector. When tracking several targets many observations are available to estimate the shared influence, improving the performance, and additionally, it is possible to track slowly changing influences over time.

To estimate the influence functions, they are in turn modelled as sparse Gaussian processes using the fully independent conditional (FIC) approximation [14]. The function values are assumed to be independent conditioned on a number of inducing variables corresponding to the function values at a set of inducing inputs. The inducing variables eliminate the need to consider all influence function values simultaneously, which enables a recursive inference method. The inducing inputs are selected prior to the estimation as a grid over the region of operation in the input space. With this model, each inducing variable reduces to a state augmented to the joint state-space model of all dynamical systems. Inference in this model is then performed using an extended Kalman filter (EKF) [15], due to the nonlinearity caused by the dependence of the input on the state.

The advantages of the proposed method, mainly as a re- 
sult of the sparse GP approximation with inducing variables, compared to state-of-the-art are that

- inference and learning for dynamical systems are integrated into a single framework;

- the complexity does not increase with larger datasets;

- learning is performed recursively online for the whole or part of a system; and

- time-varying influences can be modelled.

Due to the large dimensionality of the augmented state space and the need to maintain cross-covariances, the computational complexity can be high. To mitigate this problem, and allow for real-time implementations, possible approximations are discussed.

System identification using Gaussian process priors on the transition and measurement functions are studied in, e.g., [16], [17] and [18], using various approaches for estimating hyperparameters and latent variables. However, these methods do not directly extend to online learning, which is considered in this paper.

Several sparse approximations of Gaussian processes have been proposed in the last decades, see [14] for an overview as well as [19]. The advantage of sparse approximations is that computational and memory complexity is reduced. A model that also allows for sequential estimation is proposed in [20] and a similar approach is presented in [21]. The latter is expanded into a full framework in [22] for online identification of ARX models, taking noisy inputs into consideration. The method in [20] was successfully used in [23] with direct measurements of inducing variables augmented to the states, as opposed to the indirect measurements considered here. A similar approach using direct measurements is also described in [24]. These methods together with the descriptions in [14] work as the basis for the approach to inference and learning in state-space models proposed in this paper.

The outline of the paper is as follows. Models of dynamic systems with an unknown influence are derived step-by-step in Sec. II. Estimation using an EKF is derived in Sec. III and various implementation considerations are discussed in Sec. IV. A simulated scenario and an application in tracking is presented in Sec. V to demonstrate the proposed method.

\section{Modelling of the Influence}

The proposed model and some variations are derived stepby-step in the following sections. First, linear Gaussian models with posterior distributions that can be computed analytically, since the input to the function is a known input to the model, are presented. Then an extension to a more useful nonlinear model where the input to the function depends on the state is provided.

\section{A. Dynamic System with an Unknown Influence}

A linear Gaussian model of a dynamic system is considered,

$$
\begin{array}{lll}
\mathbf{x}_{0} \sim \mathcal{N}\left(\overline{\mathbf{x}}_{0}, \mathbf{P}_{0}\right), & & \\
\mathbf{x}_{k}=\mathbf{A}_{k} \mathbf{x}_{k-1}+\mathbf{B}_{k} \mathbf{f}_{k-1}+\mathbf{v}_{k}, & & k=1, \ldots, K \\
\mathbf{y}_{k}=\mathbf{C}_{k} \mathbf{x}_{k}+\mathbf{e}_{k}, & k=1, \ldots, K
\end{array}
$$

$$
\begin{aligned}
& \mathbf{f}_{k}=\left(f^{1}\left(\mathbf{z}_{k}^{f}\right), \ldots, f^{J}\left(\mathbf{z}_{k}^{f}\right)\right)^{T} \\
& f^{j}(\mathbf{z}) \sim \mathcal{G} \mathcal{P}\left(0, K\left(\mathbf{z}, \mathbf{z}^{\prime}\right)\right), \quad j=1, \ldots, J
\end{aligned}
$$

where $\mathbf{x}_{k} \in \mathbb{R}^{n}, \mathbf{y}_{k} \in \mathbb{R}^{m}$ and $\mathbf{z}_{k}^{f} \in \mathbb{R}^{p}$ are the state, the observation and the input, respectively, at time $k$. The input is known but influences the system in an unknown way, which is modelled by independent functions with Gaussian process [13] priors, denoted $\mathcal{G P}\left(\mu(\mathbf{z}), K\left(\mathbf{z}, \mathbf{z}^{\prime}\right)\right)$, where $\mu(\mathbf{z})$ is the mean and the kernel $K\left(\mathbf{z}, \mathbf{z}^{\prime}\right)$ encodes the covariance. The noise variables are defined as $\mathbf{v}_{k} \sim \mathcal{N}\left(\mathbf{0}, \mathbf{Q}_{k}\right)$ and $\mathbf{e}_{k} \sim \mathcal{N}\left(\mathbf{0}, \mathbf{R}_{k}\right)$, where $\mathcal{N}(\boldsymbol{\mu}, \boldsymbol{\Sigma})$ denotes the multivariate Gaussian distribution with mean $\boldsymbol{\mu}$ and covariance $\boldsymbol{\Sigma}$.

The notation $\mathbf{K}_{a b}$ will be used to denote the covariance matrix generated by the kernel $K\left(\mathbf{z}, \mathbf{z}^{\prime}\right)$ such that its elements are $\left[\mathbf{K}_{a b}\right]_{i l}=K\left(\mathbf{z}_{i}^{a}, \mathbf{z}_{l}^{b}\right)$, covering the range of indices $i$ and $l$ for the inputs. Similarly, the notation $\mathbf{K}_{a}$. $(\mathbf{z})$ will be used to denote the column vector of cross-covariances such that $\left[\mathbf{K}_{a} \cdot(\mathbf{z})\right]_{i}=K\left(\mathbf{z}_{i}^{a}, \mathbf{z}\right)$, and $\mathbf{K}_{\cdot a}(\mathbf{z})=\mathbf{K}_{a .}^{T}(\mathbf{z})$. Another shorthand is $\mathbf{K}_{a b}^{i}=\mathbf{K}_{\cdot b}\left(\mathbf{z}_{i}^{a}\right)$. Further, the notation $\tilde{\mathbf{A}}=\mathbf{A} \otimes \mathbf{I}_{J}$ will be used, where $\otimes$ denotes the Kronecker product and $\mathbf{I}_{a}$ denotes the $a \times a$ identity matrix.

Using the notation $\mathcal{F}=\left(\mathbf{f}_{0}^{T}, \ldots, \mathbf{f}_{K-1}^{T}\right)^{T}$, (1d)-(1e) can be replaced by $\mathcal{F} \sim \mathcal{N}\left(\mathbf{0}, \tilde{\mathbf{K}}_{f f}\right)$. It can then be seen that the model in (1) is a linear Gaussian state-space model for which the posterior smoothing distribution can be computed analytically [25].

\section{B. Approximation of the Influence Function}

By restricting the influence function kernel to use inducing variables, a recursive solution to the posterior filter distribution can be obtained [21].

The inducing inputs are fixed at $\mathbf{z}_{l}^{u}$, for $l=1, \ldots, L$ and the influence function values are given by the vector $\mathbf{u}_{l}=\left(f^{1}\left(\mathbf{z}_{l}^{u}\right), \ldots, f^{J}\left(\mathbf{z}_{l}^{u}\right)\right)^{T}$ where $\mathcal{U}=\left(\mathbf{u}_{1}^{T}, \ldots, \mathbf{u}_{L}^{T}\right)^{T}$. In [20] and [22] the inducing variables are used as states. Inspired by [14], $\mathcal{W}=\left(\mathbf{w}_{1}^{T}, \ldots, \mathbf{w}_{L}^{T}\right)^{T}=\tilde{\mathbf{K}}_{u u}^{-1} \mathcal{U}$ will be used as the states instead. The fully independent conditional (FIC) approximation [14] results in

$$
\left(\begin{array}{c}
\mathcal{F} \\
\mathcal{W}
\end{array}\right) \sim \mathcal{N}\left(\mathbf{0},\left(\begin{array}{cc}
\mathbf{Q}_{f f}+\boldsymbol{\Lambda} & \mathbf{K}_{f u} \mathbf{K}_{u u}^{-1} \\
\mathbf{K}_{u u}^{-1} \mathbf{K}_{u f} & \mathbf{K}_{u u}^{-1}
\end{array}\right) \otimes \mathbf{I}_{J}\right),
$$

where $\mathbf{Q}_{a b}=\mathbf{K}_{a u} \mathbf{K}_{u u}^{-1} \mathbf{K}_{u b}$ and $\boldsymbol{\Lambda}=\operatorname{diag}\left(\mathbf{K}_{f f}-\mathbf{Q}_{f f}\right)$. The model can be rewritten, see Appendix $\mathrm{C}$, in the form

$$
\begin{aligned}
\mathbf{x}_{0} & \sim \mathcal{N}\left(\overline{\mathbf{x}}_{0}, \mathbf{P}_{0}\right), \\
\mathbf{x}_{k} & =\mathbf{A}_{k} \mathbf{x}_{k-1}+\mathbf{B}_{k}\left(\tilde{\mathbf{K}}_{f u}^{k-1} \mathcal{W}+\mathbf{v}_{k}^{f}\right)+\mathbf{v}_{k}, \\
\mathbf{y}_{k} & =\mathbf{C}_{k} \mathbf{x}_{k}+\mathbf{e}_{k}, \\
\mathcal{W} & \sim \mathcal{N}\left(\mathbf{0}, \tilde{\mathbf{K}}_{u u}^{-1}\right),
\end{aligned}
$$

where $\mathbf{v}_{k}^{f} \sim \mathcal{N}\left(\mathbf{0}, \tilde{\Lambda}_{k}\right)$ and $\Lambda_{k}=[\boldsymbol{\Lambda}]_{k k}$. In case the subset of regressors (SOR) approximation [14] is used let $\boldsymbol{\Lambda}=\mathbf{0}$, effectively eliminating $\mathbf{v}_{k}^{f}$ from the model. The model can be extended by including a prior mean $\overline{\mathcal{W}}_{0}=\tilde{\mathbf{K}}_{u u}^{-1} \overline{\mathcal{U}}_{0}$ of the function in (3d). 


\section{Time-Varying Influence}

As noted in [23] and [26] the model in (3) allows the influence to be time-varying with a slight extension,

$$
\begin{aligned}
\mathbf{x}_{0} & \sim \mathcal{N}\left(\overline{\mathbf{x}}_{0}, \mathbf{P}_{0}\right), \\
\mathbf{x}_{k} & =\mathbf{A}_{k} \mathbf{x}_{k-1}+\mathbf{B}_{k}\left(\tilde{\mathbf{K}}_{f u}^{k-1} \mathcal{W}_{k-1}+\mathbf{v}_{k}^{f}\right)+\mathbf{v}_{k}, \\
\mathbf{y}_{k} & =\mathbf{C}_{k} \mathbf{x}_{k}+\mathbf{e}_{k}, \\
\mathcal{W}_{0} & \sim \mathcal{N}\left(\overline{\mathcal{W}}_{0}, \tilde{\mathbf{K}}_{u u}^{-1}\right), \\
\mathcal{W}_{k} & =\mathbf{G}_{k} \mathcal{W}_{k-1}+\mathbf{v}_{k}^{w},
\end{aligned}
$$

where $\mathbf{v}_{k}^{w} \sim \mathcal{N}\left(\boldsymbol{\mu}_{k}, \boldsymbol{\Sigma}_{k}\right)$. Influences, such as wind and currents, can thus be modelled to vary with time by introducing uncertainty in the form of process noise.

There are several options for $\mathbf{G}, \boldsymbol{\mu}_{k}$ and $\boldsymbol{\Sigma}_{k}$ that might be useful. In both [23] and [26] the transition matrix, mean and covariance are chosen as

$$
\begin{aligned}
\mathbf{G} & =e^{-\alpha T} \mathbf{I}, \\
\boldsymbol{\mu}_{k} & =\left(1-e^{-\alpha T}\right) \overline{\mathcal{W}}_{0}, \\
\boldsymbol{\Sigma}_{k} & =\left(1-e^{-2 \alpha T}\right) \tilde{\mathbf{K}}_{u u}^{-1},
\end{aligned}
$$

where $T$ is the sampling time and $\alpha$ is a forgetting factor determining the rate of convergence back to the prior distribution $\mathcal{W}_{0}$.

Another option is simply to let $\mathbf{G}=\mathbf{I}, \boldsymbol{\mu}_{k}=\mathbf{0}$ and $\boldsymbol{\Sigma}_{k}=\alpha \tilde{\mathbf{K}}_{u u}^{-1}$. The process will not converge to a stationary distribution, but the mean will remain unchanged, which might be desirable in some situations.

\section{Multiple Systems with Shared Influence}

In many scenarios multiple systems exhibit similar behaviour due to common influences from the surrounding environment, e.g. by wind, currents, paths and obstacles. Estimation of the common influence and the predictions of the systems are then improved by observing multiple systems.

The model in (4) is extended as

$$
\begin{aligned}
\mathbf{x}_{0}^{i} & \sim \mathcal{N}\left(\overline{\mathbf{x}}_{0}^{i}, \mathbf{P}_{0}^{i}\right), \\
\mathbf{x}_{k}^{i} & =\mathbf{A}_{k} \mathbf{x}_{k-1}^{i}+\mathbf{B}_{k}\left(\tilde{\mathbf{K}}_{i u}^{k-1} \mathcal{W}_{k-1}+\mathbf{v}_{k}^{i f}\right)+\mathbf{v}_{k}^{i}, \\
\mathbf{y}_{k}^{i} & =\mathbf{C}_{k} \mathbf{x}_{k}^{i}+\mathbf{e}_{k}^{i}, \\
\mathcal{W}_{0} & \sim \mathcal{N}\left(\overline{\mathcal{W}}_{0}, \tilde{\mathbf{K}}_{u u}^{-1}\right), \\
\mathcal{W}_{k} & =\mathbf{G}_{k} \mathcal{W}_{k-1}+\mathbf{v}_{k}^{w},
\end{aligned}
$$

where $\mathbf{z}_{k}^{i}$ is the input for process $i=1, \ldots, I$ and $\mathbf{v}_{k}^{i} \sim$ $\mathcal{N}\left(\mathbf{0}, \mathbf{Q}_{k}^{i}\right), \mathbf{e}_{k}^{i} \sim \mathcal{N}\left(\mathbf{0}, \mathbf{R}_{k}^{i}\right)$ and $\mathbf{v}_{k}^{i f} \sim \mathcal{N}\left(\mathbf{0}, \tilde{\Lambda}_{k}^{i}\right)$ are noise variables with $\Lambda_{k}^{i}=\left[\mathbf{K}_{i i}-\mathbf{Q}_{i i}\right]_{k k}$. In the sequel the notation $\mathcal{X}_{k}=\left(\mathbf{x}_{k}^{1 T}, \ldots, \mathbf{x}_{k}^{I T}\right)^{T}$ and $\mathcal{Y}_{k}=\left(\mathbf{y}_{k}^{1 T}, \ldots, \mathbf{y}_{k}^{I T}\right)^{T}$ will be used. Although the processes are independent conditioned on the function, a dependence is introduced between the processes by the uncertainty of the function.

\section{E. State-Dependent Influence}

In many cases the inputs to the influence functions are not known inputs to the system, but depend on the state. The inputs are therefore modified to depend on the state as $\mathbf{z}_{k-1}^{i}=\mathbf{D}_{k} \mathbf{x}_{k-1}^{i}$. This model will be considered in the remainder of the paper and is given by

$$
\begin{aligned}
\mathbf{x}_{0}^{i} \sim & \mathcal{N}\left(\overline{\mathbf{x}}_{0}^{i}, \mathbf{P}_{0}^{i}\right), \\
\mathbf{x}_{k}^{i}= & \mathbf{A}_{k} \mathbf{x}_{k-1}^{i}+\mathbf{B}_{k}\left(\tilde{\mathbf{K}}_{\cdot u}\left(\mathbf{D}_{k} \mathbf{x}_{k-1}^{i}\right) \mathcal{W}_{k-1}+\right. \\
& \left.\mathbf{v}_{k}^{i f}\left(\mathbf{D}_{k} \mathbf{x}_{k-1}^{i}\right)\right)+\mathbf{v}_{k}^{i} \\
\mathbf{y}_{k}^{i}= & \mathbf{C}_{k} \mathbf{x}_{k}^{i}+\mathbf{e}_{k}^{i} \\
\mathcal{W}_{0} \sim & \mathcal{N}\left(\overline{\mathcal{W}}_{0}, \tilde{\mathbf{K}}_{u u}^{-1}\right) \\
\mathcal{W}_{k}= & \mathbf{G}_{k} \mathcal{W}_{k-1}+\mathbf{v}_{k}^{w}
\end{aligned}
$$

which is no longer linear. Note that $\mathbf{v}_{k}^{i f}(\mathbf{z}) \sim \mathcal{N}(\mathbf{0}, \tilde{\Lambda}(\mathbf{z}))$, where $\Lambda(\mathbf{z})=K(\mathbf{z}, \mathbf{z})-\mathbf{K}_{\cdot u}(\mathbf{z}) \mathbf{K}_{u u}^{-1} \mathbf{K}_{u} \cdot(\mathbf{z})=K(\mathbf{z}, \mathbf{z})-$ $\sum_{l=1}^{L} \sum_{l^{\prime}=1}^{L} K\left(\mathbf{z}, \mathbf{z}_{l}^{u}\right)\left[\mathbf{K}_{u u}^{-1}\right]_{l l^{\prime}} K\left(\mathbf{z}_{l^{\prime}}^{u}, \mathbf{z}\right)$. Letting $i=1$ a model for a single dynamical system is obtained as a special case.

\section{F. Covariance Function for the Influence}

The choice of covariance function $K\left(\mathbf{z}, \mathbf{z}^{\prime}\right)$ is important as it encodes the assumptions made on the function to be learnt [23]. There are many covariance functions to consider, see e.g. [13], which additionally depend on hyperparameters that need to be selected carefully. The hyperparameters are either selected manually using expert knowledge or are learned from the data [13], [20], [22], [27], where the latter is often preferred, but results in additional steps that may be complicated depending on the problem at hand.

For simplicity, the squared-exponential kernel, given by

$$
K\left(\mathbf{z}, \mathbf{z}^{\prime}\right)=\sigma^{2} \exp \left(-\frac{1}{2 \theta^{2}}\left\|\mathbf{z}-\mathbf{z}^{\prime}\right\|^{2}\right),
$$

is used in the remainder of the paper and the hyperparameters are selected manually. The hyperparameter $\theta$ works as a length scale for the covariance function inputs, modelling the smoothness of the function, and $\sigma^{2}$ determines the variance of the function, modelling the belief in the prior mean. The estimation approach considered in this paper also requires the derivative of the covariance function, which is given by

$$
\begin{aligned}
\mathbf{K}_{\mathbf{z}^{\prime}}\left(\mathbf{z}, \mathbf{z}^{\prime}\right) & \triangleq \frac{\partial}{\partial \mathbf{z}^{\prime}} K\left(\mathbf{z}, \mathbf{z}^{\prime}\right)=\frac{K\left(\mathbf{z}, \mathbf{z}^{\prime}\right)}{\theta^{2}}\left(\mathbf{z}-\mathbf{z}^{\prime}\right)^{T} \\
& =-\frac{\partial}{\partial \mathbf{z}} K\left(\mathbf{z}, \mathbf{z}^{\prime}\right) \triangleq-\mathbf{K}_{\mathbf{z}}\left(\mathbf{z}, \mathbf{z}^{\prime}\right),
\end{aligned}
$$

where the numerator layout notation, $\frac{\partial}{\partial \mathbf{b}} \mathbf{a}=\left[\frac{\partial \mathbf{a}}{\partial b_{1}}, \ldots, \frac{\partial \mathbf{a}}{\partial b_{n}}\right]$, is used to obtain Jacobians.

\section{ESTIMATION}

The posterior smoothing distribution for the states is derived for the model in Sec. II-A. For the remaining linear models recursive solutions are obtained using Kalman filters [28]. The posterior distribution for the last model is approximated using an extended Kalman filter (EKF) [15] as derived below. 


\section{A. Posterior Smoothing Distribution}

The model in Sec. II-A is linear and Gaussian and its posterior can be derived analytically. Let $\mathcal{X}=\left(\mathbf{x}_{0}^{T}, \ldots, \mathbf{x}_{K}^{T}\right)^{T}$, $\mathcal{F}=\left(\mathbf{f}_{1}^{T}, \ldots, \mathbf{f}_{K}^{T}\right)^{T}$ and $\mathcal{Y}=\left(\mathbf{y}_{1}^{T}, \ldots, \mathbf{y}_{K}^{T}\right)^{T}$. Replacing (1d)(1e) with $\mathcal{F} \sim \mathcal{N}\left(\mathbf{0}, \tilde{\mathbf{K}}_{f f}\right)$ and moving knowns to the left and unknowns to the right allows a representation of (1) on the form

$$
\overline{\mathbf{y}}=\overline{\mathbf{H}}\left(\begin{array}{l}
\mathcal{X} \\
\mathcal{F}
\end{array}\right)+\overline{\mathbf{e}}
$$

where $\overline{\mathbf{y}}=\left(\overline{\mathbf{x}}_{0}^{T}, \mathbf{0}^{T}, \mathcal{Y}^{T}, \mathbf{0}^{T}\right)^{T}, \operatorname{cov}(\overline{\mathbf{e}})=\overline{\mathbf{R}}$,

$$
\overline{\mathbf{R}}=\operatorname{diag}\left(\mathbf{P}_{0}, \mathbf{Q}_{1}, \ldots, \mathbf{Q}_{K}, \mathbf{R}_{1}, \ldots, \mathbf{R}_{K}, \tilde{\mathbf{K}}_{f f}\right),
$$

and the sparse matrix $\overline{\mathbf{H}}$ follows naturally. Using the chain rule the joint probability distribution is expressed in terms of the observations, dynamics and GP prior as

$$
\begin{array}{r}
p(\mathcal{Y}, \mathcal{X}, \mathcal{F})=p(\mathcal{Y} \mid \mathcal{X}) p(\mathcal{X} \mid \mathcal{F}) p(\mathcal{F}) \\
=\prod_{k=1}^{K} \mathcal{N}\left(\mathbf{y}_{k} ; \mathbf{C}_{k} \mathbf{x}_{k}, \mathbf{R}_{k}\right) \times \mathcal{N}\left(\mathbf{x}_{0} ; \overline{\mathbf{x}}_{0}, \mathbf{P}_{0}\right) \times \\
\prod_{k=1}^{K} \mathcal{N}\left(\mathbf{x}_{k} ; \mathbf{A}_{k} \mathbf{x}_{k-1}+\mathbf{B}_{k} \mathbf{f}_{k-1}, \mathbf{Q}_{k}\right) \times \\
\mathcal{N}\left(\mathcal{F} ; \mathbf{0}, \tilde{\mathbf{K}}_{f f}\right)=\mathcal{N}\left(\overline{\mathbf{y}} ; \overline{\mathbf{H}}\left(\begin{array}{c}
\mathcal{X} \\
\mathcal{F}
\end{array}\right), \overline{\mathbf{R}}\right) .
\end{array}
$$

The joint posterior distribution between the states and the unknown function is given by $p(\mathcal{X}, \mathcal{F} \mid \mathcal{Y}) \propto p(\mathcal{Y}, \mathcal{X}, \mathcal{F})$. Manipulating the product of Gaussians using Proposition 1 in Appendix A results in the smoothing posterior

$$
\left(\begin{array}{l}
\mathcal{X} \\
\mathcal{F}
\end{array}\right) \mid \mathcal{Y} \sim \mathcal{N}\left(\left(\overline{\mathbf{H}}^{T} \overline{\mathbf{R}}^{-1} \overline{\mathbf{H}}\right)^{-1} \overline{\mathbf{H}}^{T} \overline{\mathbf{R}}^{-1} \overline{\mathbf{y}}, \overline{\mathbf{H}}^{T} \overline{\mathbf{R}}^{-1} \overline{\mathbf{H}}\right) .
$$

Similar calculations can be used to obtain the posterior distribution for the remaining linear models. However, their structures lend themselves to recursive estimation algorithms that are more efficient since they do not require the knowledge of all inputs in advance.

\section{B. Recursive Estimation Using the Extended Kalman Filter}

The EKF [15] is an algorithm that recursively assumes a Gaussian approximation for the prediction and filter distributions [25], denoted by

$$
\left(\begin{array}{c}
\mathcal{X}_{k} \\
\mathcal{W}_{k}
\end{array}\right) \mid \mathcal{Y}_{1: k-1} \sim \mathcal{N}\left(\left(\begin{array}{c}
\overline{\mathcal{X}}_{k} \\
\overline{\mathcal{W}}_{k}
\end{array}\right),\left(\begin{array}{cc}
\overline{\mathbf{P}}_{x, k} & \overline{\mathbf{P}}_{w x, k} \\
\overline{\mathbf{P}}_{w x, k}^{T} & \overline{\mathbf{P}}_{w, k}
\end{array}\right)\right),
$$

and

$$
\left(\begin{array}{c}
\mathcal{X}_{k} \\
\mathcal{W}_{k}
\end{array}\right) \mid \mathcal{Y}_{1: k} \sim \mathcal{N}\left(\left(\begin{array}{c}
\hat{\mathcal{X}}_{k} \\
\hat{\mathcal{W}}_{k}
\end{array}\right),\left(\begin{array}{cc}
\mathbf{P}_{x, k} & \mathbf{P}_{w x, k} \\
\mathbf{P}_{w x, k}^{T} & \mathbf{P}_{w, k}
\end{array}\right)\right),
$$

respectively, where the notation $\overline{\mathcal{X}}_{k}=\left(\overline{\mathbf{x}}_{k}^{1 T}, \ldots, \overline{\mathbf{x}}_{k}^{I T}\right)^{T}$ and $\hat{\mathcal{X}}_{k}=\left(\hat{\mathbf{x}}_{k}^{1 T}, \ldots, \hat{\mathbf{x}}_{k}^{I T}\right)^{T}$ is used and $\mathcal{Y}_{1: k}$ denotes all measurements up to time step $k$. Each iteration consists of a prediction and a correction step, in which the transition and observation functions are linearised, respectively.
1) Prediction: The transition function for the dynamical states in (7b) is linear in the inducing states and the process noise, but nonlinear in the dynamical states. The Jacobian with regards to the dynamical states is given by

$$
\begin{gathered}
\frac{\partial \mathbf{x}_{k}^{i}}{\partial \mathbf{x}_{k-1}^{i}}=\mathbf{A}_{k}+\mathbf{B}_{k}\left(\sum_{l=1}^{L} \mathbf{w}_{k-1}^{l} \mathbf{K}_{\mathbf{z}}\left(\mathbf{D}_{k} \mathbf{x}_{k-1}^{i}, \mathbf{z}_{l}^{u}\right)+\right. \\
\left.\mathbf{v}_{k}^{i f}\left(\mathbf{D}_{k} \mathbf{x}_{k-1}^{i}\right) \frac{\Lambda_{\mathbf{z}}\left(\mathbf{D}_{k} \mathbf{x}_{k-1}^{i}\right)}{2 \Lambda\left(\mathbf{D}_{k} \mathbf{x}_{k-1}^{i}\right)}\right) \mathbf{D}_{k},
\end{gathered}
$$

using Proposition 2 in Appendix B, which is used to define

$$
\begin{aligned}
\mathbf{F}_{x}^{i} & \left.\triangleq \frac{\partial \mathbf{x}_{k}^{i}}{\partial \mathbf{x}_{k-1}^{i}}\right|_{\mathbf{x}_{k-1}^{i}=\hat{\mathbf{x}}_{k-1}^{i}, \mathcal{W}_{k-1}=\hat{\mathcal{W}}_{k-1}, \mathbf{v}_{k}^{i f}\left(\mathbf{D}_{k} \mathbf{x}_{k-1}^{i}\right)=\mathbf{0}} \\
& =\mathbf{A}_{k}+\mathbf{B}_{k}\left(\sum_{l=1}^{L} \hat{\mathbf{w}}_{k-1}^{l} \mathbf{K}_{\mathbf{z}}\left(\mathbf{D}_{k} \hat{\mathbf{x}}_{k-1}^{i}, \mathbf{z}_{l}^{u}\right)\right) \mathbf{D}_{k}, \\
\mathbf{F}_{w}^{i} & \left.\triangleq \frac{\partial \mathbf{x}_{k}^{i}}{\partial \mathcal{W}_{k-1}}\right|_{\mathbf{x}_{k-1}^{i}=\hat{\mathbf{x}}_{k-1}^{i}, \mathcal{W}_{k-1}=\hat{\mathcal{W}}_{k-1}} \\
& =\mathbf{B}_{k} \tilde{\mathbf{K}}_{\cdot u}\left(\mathbf{D}_{k} \hat{\mathbf{x}}_{k-1}^{i}\right),
\end{aligned}
$$

where the time index $k$ is left out for brevity. The system function for the inducing states in (7e) is linear in all states and the noise. The prediction step in the extended Kalman filter is then given by

$$
\begin{aligned}
\overline{\mathbf{x}}_{k}^{i}= & \mathbf{A}_{k} \hat{\mathbf{x}}_{k-1}^{i}+\mathbf{B}_{k} \tilde{\mathbf{K}}_{\cdot u}\left(\mathbf{D}_{k} \hat{\mathbf{x}}_{k-1}^{i}\right) \hat{\mathcal{W}}_{k-1}, \\
\overline{\mathcal{W}}_{k}= & \mathbf{G}_{k} \hat{\mathcal{W}}_{k-1}+\boldsymbol{\mu}_{k}, \\
\overline{\mathbf{P}}_{x, k}^{i, n}= & \mathbf{F}_{x}^{i} \mathbf{P}_{x, k-1}^{i, n}\left(\mathbf{F}_{x}^{n}\right)^{T}+\mathbf{F}_{w}^{i} \mathbf{P}_{w x, k-1}^{\cdot, n}\left(\mathbf{F}_{x}^{n}\right)^{T}+ \\
& \mathbf{F}_{x}^{i}\left(\mathbf{F}_{w}^{n} \mathbf{P}_{w x, k-1}^{,, i}\right)^{T}+\mathbf{F}_{w}^{i} \mathbf{P}_{w, k-1}\left(\mathbf{F}_{w}^{n}\right)^{T}+ \\
& +\left(\mathbf{B}_{k} \Lambda\left(\mathbf{D}_{k} \hat{\mathbf{x}}_{k-1}^{i}\right)\left(\mathbf{B}_{k}\right)^{T}+\mathbf{Q}_{k}^{i}\right) \delta_{i n}, \\
\overline{\mathbf{P}}_{w x, k}^{j, i}= & \mathbf{G}^{j, \cdot} \mathbf{P}_{w x, k-1}^{, i,}\left(\mathbf{F}_{x}^{i}\right)^{T}+\mathbf{G}^{j, \cdot} \mathbf{P}_{w, k-1}\left(\mathbf{F}_{w}^{i}\right)^{T}, \\
\overline{\mathbf{P}}_{w, k}^{j, l}= & \mathbf{G}^{j,} \mathbf{P}_{w, k-1}\left(\mathbf{G}^{l, \cdot}\right)^{T}+\mathbf{\Sigma}_{k}^{j, l},
\end{aligned}
$$

where $i=1, \ldots, I$ and $n=1, \ldots, I$ index submatrices corresponding to dynamic states, $j=1, \ldots, L$ and $l=1, \ldots, L$ index submatrices corresponding to inducing states and $\delta_{i n}$ is the Kronecker delta.

2) Correction: The correction step in the extended Kalman filter is given for a single observation by

$$
\begin{aligned}
\mathbf{S}_{k}^{i} & \triangleq \mathbf{C}_{k} \overline{\mathbf{P}}_{x, k}^{i, i} \mathbf{C}_{k}^{T}+\mathbf{R}_{k}^{i}, \\
\mathbf{G}_{x, k}^{i} & \triangleq \overline{\mathbf{P}}_{x, k}^{\cdot, i} \mathbf{C}_{k}^{T}\left(\mathbf{S}_{k}^{i}\right)^{-1}, \\
\mathbf{G}_{w, k}^{i} & \triangleq \overline{\mathbf{P}}_{w x, k}^{\cdot, i} \mathbf{C}_{k}^{T}\left(\mathbf{S}_{k}^{i}\right)^{-1}, \\
\hat{\mathcal{X}}_{k} & =\overline{\mathcal{X}}_{k}+\mathbf{G}_{x, k}^{i}\left(\mathbf{y}_{k}^{i}-\mathbf{C}_{k} \overline{\mathbf{x}}_{k}^{i}\right), \\
\hat{\mathcal{W}}_{k} & =\overline{\mathcal{W}}_{k}+\mathbf{G}_{w, k}^{i}\left(\mathbf{y}_{k}^{i}-\mathbf{C}_{k} \overline{\mathbf{x}}_{k}^{i}\right), \\
\mathbf{P}_{x, k} & =\overline{\mathbf{P}}_{x, k}-\mathbf{G}_{x, k}^{i} \mathbf{S}_{k}^{i}\left(\mathbf{G}_{x, k}^{i}\right)^{T}, \\
\mathbf{P}_{w x, k} & =\overline{\mathbf{P}}_{w x, k}-\mathbf{G}_{w, k}^{i} \mathbf{S}_{k}^{i}\left(\mathbf{G}_{x, k}^{i}\right)^{T}, \\
\mathbf{P}_{w, k} & =\overline{\mathbf{P}}_{w, k}-\mathbf{G}_{w, k}^{i} \mathbf{S}_{k}^{i}\left(\mathbf{G}_{w, k}^{i}\right)^{T} .
\end{aligned}
$$

The correction is applied for each observation in turn, using the filter distribution from the previous correction as the prediction distribution. 


\section{IMPLEMENTATION CONSIDERATIONS}

Direct implementation of the equations in the previous steps may be expensive in the general case, even for a moderate grid size and a moderate number of dynamical systems since the cross-covariances between all states are maintained. However, the potential improvement over models disregarding the influence lies in the cross-covariances. Methods are therefore proposed in this section to only maintain and update significant cross-covariances.

\section{A. Covariance Function Truncation}

By expanding the matrix multiplications in (17c) it is noted that all terms that introduce cross-covariances in the prediction step directly scale with the kernel value. The contribution to the cross-covariances by terms where the kernel value is small is negligible. By truncating the normalised kernel,

$$
\tilde{K}\left(\mathbf{x}, \mathbf{x}^{\prime}\right)= \begin{cases}K\left(\mathbf{x}, \mathbf{x}^{\prime}\right), & \sigma^{2} K\left(\mathbf{x}, \mathbf{x}^{\prime}\right)<\epsilon_{K}, \\ 0, & \text { otherwise, }\end{cases}
$$

the number of terms in the computation decreases and small cross-covariances reduce to exactly zero. The risk is that the covariance matrix loses positive-definiteness [29], so the threshold $\epsilon_{K}$ must be set low enough. This improves the performance with negligible effect to the results if the length parameter is relatively small and $\epsilon_{K}$ is appropriately chosen.

\section{B. Inducing Input Grid}

The number of inducing variables, and thus the required memory and computations, directly depends on the resolution of the inducing input grid. The resolution is a trade-off between the sparsity of the function and the performance. If the resolution is too fine, the covariance between the inducing variables is large and less information is stored per variable. If it is too coarse, the inducing variables struggle to represent the underlying function.

Since the inputs scale with the length parameter $\theta$ in (8) the distance between the inducing inputs is selected relative to this parameter. Simple tests were performed for one dimensional inputs comparing the Frobenius norm of the covariance matrices $\mathbf{K}_{f f}$ and $\mathbf{Q}_{f f}$, as $\left\|\mathbf{K}_{f f}-\mathbf{Q}_{f f}\right\|_{F} /\left\|\mathbf{K}_{f f}\right\|_{F}$, for $\mathbf{z}_{l}^{u}$ gridded relative to the length parameter and random $\mathbf{z}_{k}^{f}$. They indicate that the error is insignificant for a grid distance less than $\theta / 3$, around $1 \%$ for a distance around $\theta$ and growing fast for distances greater than $\theta$. As a trade-off between performance and computational burden, the grid distance is proposed to be chosen in the interval $[\theta / 3, \theta]$.

Remark. One might contemplate allowing inducing points to be added, removed or moved, as suggested in [22]. This is unfortunately not as straightforward as it might seem. Let $\mathbf{z}_{\hat{u}}$ denote added inducing inputs and $\mathbf{z}_{\bar{u}}$ the augmented set of original and added inducing inputs. The weights for the new set of inducing variables are given by

$$
\begin{aligned}
\overline{\mathcal{W}} & =\tilde{\mathbf{K}}_{\bar{u} \bar{u}}^{-1} \tilde{\mathbf{K}}_{\bar{u} u} \mathcal{W} \\
& =\left(\begin{array}{ll}
\mathbf{K}_{u u} & \mathbf{K}_{u \hat{u}} \\
\mathbf{K}_{\hat{u} u} & \mathbf{K}_{\hat{u} \hat{u}}
\end{array}\right)^{-1}\left(\begin{array}{l}
\mathbf{K}_{u u} \\
\mathbf{K}_{\hat{u} u}
\end{array}\right) \mathcal{W}=\left(\begin{array}{l}
\mathbf{I} \\
\mathbf{0}
\end{array}\right) \mathcal{W} .
\end{aligned}
$$

The new inducing variables are identically zero, i.e., degenerate Gaussian with zero mean and zero variance, and will therefore not improve the flexibility of the function estimate. Adding new observations will not improve the situation. Removing inducing variables reduces the flexibility of the function estimate. Consequently, modifying the set of inducing variables by simply adding and removing variables will eventually result in a function estimate that approaches identically zero. The set of inducing variables is therefore assumed to be fixed.

\section{Cross-Covariance Thresholding}

Relevant cross-covariances might decrease over time, so a mechanism to threshold and effectively remove small crosscovariances would reduce the computational cost. To obtain a method that is independent of the state variances it is proposed that thresholding is performed in the correlation matrix,

$$
\mathbf{C}=\mathbf{D}^{-1} \mathbf{P D}^{-1}, \quad \mathbf{D}^{2}=\operatorname{diag}(\mathbf{P})
$$

to determine which cross-covariances to remove. However, removing cross-covariances does not guarantee that the resulting covariance matrix remains positive definite. By setting the threshold very low the risk is mitigated in practice, while still allowing almost zero cross-covariances to be disregarded in computations. To handle a larger threshold while still guaranteeing positive definiteness, the covariance matrix needs to be compensated for the removed cross-covariances.

We propose a method based on the idea that the sum of a positive-definite matrix and a positive-semi-definite matrix is positive definite. For the removal of a single cross-covariance $\mathbf{P}_{i j}=\mathbf{P}_{j i}$, the sparse approximation of the covariance matrix is computed as

$$
\tilde{\mathbf{P}}=\mathbf{P}+\tilde{\mathbf{I}}_{i j}^{T} \mathbf{E} \tilde{\mathbf{I}}_{i j},
$$

where the selector matrix is defined as $\tilde{\mathbf{I}}_{i j}\left(x_{1} \ldots x_{n}\right)^{T}=$ $\left(\begin{array}{ll}x_{i} & x_{j}\end{array}\right)^{T}$ and

$$
\mathbf{E}=\left(\begin{array}{cc}
\sqrt{\frac{\mathbf{P}_{i i}}{\mathbf{P}_{j j}}}\left|\mathbf{P}_{i j}\right| & -\mathbf{P}_{i j} \\
-\mathbf{P}_{i j} & \sqrt{\frac{\mathbf{P}_{j j}}{\mathbf{P}_{i i}}}\left|\mathbf{P}_{i j}\right|
\end{array}\right) .
$$

The two diagonal elements of $\mathbf{E}$ are chosen to achieve a rank one non-negative definite matrix such that the resulting determinant of the covariance matrix $\tilde{\mathbf{I}}_{i j} \tilde{\mathbf{P}}_{i j}^{T}$ is minimised.

\section{Gating Inputs to Determine Significant Covariances}

To further reduce the computational burden, it would be preferable to avoid computing cross-covariances, which are the major bottle-neck in the filter, in the first place.

The first difficulty is to determine which cross-covariances will be small. A simple option to determine which crosscovariances are potentially significant is to require a maximum distance, similar to a gate, weighted by the length parameter, between the function inputs of the processes.

The second difficulty is how to compensate the diagonal of the covariance matrix for the removal of a cross-covariance when its magnitude is unknown, to avoid losing positive definiteness. A simple option is to not compensate and make 
sure the minimum distance is large enough and slightly increase the process covariance. A more complicated option is to estimate the compensation by computing a subset of the cross-covariances or computing them at a lower frequency.

\section{E. Symmetry, Sparsity and Reuse Considerations}

The covariance matrix is symmetric, hence storing and updating the entire $\mathbf{P}_{x, k}$ and $\mathbf{P}_{w, k}$ matrices result in double storage and double computation. An efficient method to insert, store, access and remove individual covariances and crosscovariances would reduce the required memory and computation. Especially if the majority of the cross-covariances are close to zero. Many numerical libraries provide efficient implementations for symmetric and sparse matrices. Often it is worthwhile to intelligently select the order of computations and quantities to reuse to avoid redundancy.

\section{Evaluation}

The proposed method is evaluated and demonstrated in simulations and with experimental data.

\section{A. Velocity Field Simulation}

To demonstrate and evaluate the method proposed in Sec. III-B a tracking scenario is simulated based on the model in Sec. II-E. The underlying model for each target is given by

$$
\begin{aligned}
& \mathbf{x}_{k}=\mathbf{x}_{k-1}+\mathbf{f}\left(\mathbf{x}_{k-1}\right)+\mathbf{v}_{k}, \\
& \mathbf{y}_{j}=\mathbf{x}_{k_{j}}+\mathbf{e}_{j},
\end{aligned}
$$

where the state $\mathbf{x}_{k}$ is the position $[\mathrm{m}]$ at time step $k=$ $1, \ldots, K, \mathbf{y}_{j}$ for $j=0, \ldots\lfloor K / S\rfloor$ is an observation of the position at time step $k_{j}=j S, S$ determines time between observations and $\mathbf{f}(\mathbf{x})$ determines the velocity of a target given its location. The velocity function $\mathbf{f}(\mathbf{x})$ is shared between all targets. The observation and process noises are given by $\mathbf{e}_{j} \sim \mathcal{N}(\mathbf{0}, \mathbf{R})$ with covariance $\mathbf{R}=9 \mathbf{I}_{2}$ and $\mathbf{v}_{k} \sim \mathcal{N}(\mathbf{0}, \mathbf{Q})$ with covariance $\mathbf{Q}=q^{2} T_{s} \mathbf{I}_{2}$, respectively, where $T_{s}=1 \mathrm{~s}$ is the discretisation time. The function, shown in Fig. 1, is continuous and designed so that the targets roughly follow a path, which, e.g., could represent a racing track. A sample of 200 targets are drawn uniformly and simulated $150 \mathrm{~s}$ according to the model. The environment is assumed to be clutter free and correct associations are assumed to be given. Data is generated with the parameters $q \in\{0,0.5,2,5,20,50\}$ and $S \in\{1,2,5,10\}$ according to the configurations shown in Tab. I. A Monte Carlo simulation is performed where a first realisation is used to optimise the hyperparameters for each model and another 100 realisations are used for estimation.

The model used for estimation is

$$
\begin{aligned}
\mathbf{x}_{0}^{i} & \sim \mathcal{N}\left(\overline{\mathbf{x}}_{0}^{i}, \mathbf{P}_{0}\right), \\
\mathbf{x}_{k}^{i} & =\mathbf{x}_{k-1}^{i}+\left(\tilde{\mathbf{K}}_{\cdot u}\left(\mathbf{x}_{k-1}^{i}\right) \mathcal{W}+\mathbf{v}_{k}^{i f}\left(\mathbf{x}_{k-1}^{i}\right)\right)+\mathbf{v}_{k}^{i}, \\
\mathbf{y}_{j}^{i} & =\mathbf{x}_{k_{j}}^{i}+\mathbf{e}_{j}^{i}, \\
\mathcal{W} & \sim \mathcal{N}\left(\mathbf{0}, \tilde{\mathbf{K}}_{u u}^{-1}\right),
\end{aligned}
$$

where the function is modelled as time-invariant, index $i$ has been added to reference the target, and the covariance function

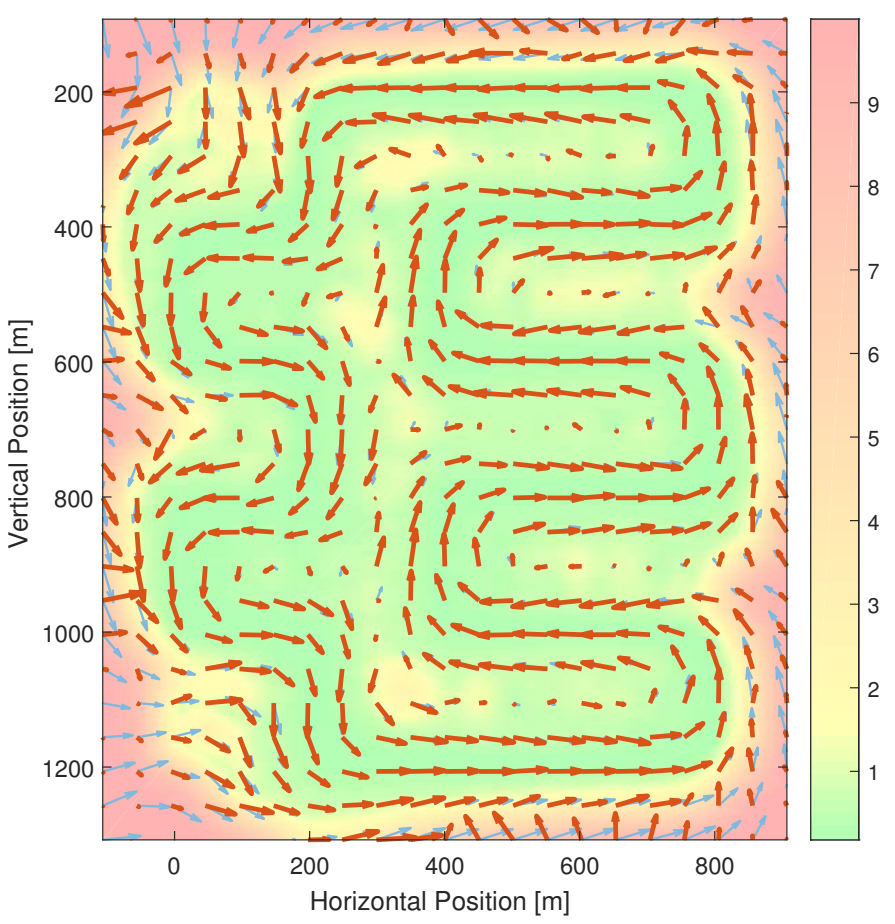

Fig. 1. The true and estimated velocity field in blue and red, respectively, for the configuration $q=0.5$ and $S=5$, where the length of the arrows corresponds to the distance travelled in $5 \mathrm{~s}$. The estimated velocity appears to converge to the true velocity along the path, but approaches the prior of zero velocity where fewer observations are available. The standard deviation of the velocity $(\mathrm{m} / \mathrm{s})$ is shown in the background.

is chosen as in (8). The inducing points are gridded in the visible part of Fig. 1 with a spacing given by $\delta$. For comparison, the near constant velocity ( $\mathrm{NCV}$ ) and acceleration ( $\mathrm{NCA}$ ) models [30] are considered, with the covariance matrix for the former chosen as

$$
\mathbf{Q}_{v}=q_{v}^{2} \cdot\left(\begin{array}{cc}
T_{s}^{3} / 3 & T_{s}^{2} / 2 \\
T_{s}^{2} / 2 & T_{s}
\end{array}\right) \otimes \mathbf{I}_{2}
$$

and the latter as

$$
\mathbf{Q}_{a}=q_{a}^{2} \cdot\left(\begin{array}{ccc}
T_{s}^{5} / 20 & T_{s}^{4} / 8 & T_{s}^{3} / 6 \\
T_{s}^{4} / 8 & T_{s}^{3} / 3 & T_{s}^{2} / 2 \\
T_{s}^{3} / 6 & T_{s}^{2} / 2 & T_{s}
\end{array}\right) \otimes \mathbf{I}_{2} .
$$

The true covariance matrix $\mathbf{R}$ is used and the process noise covariances $\mathbf{Q}_{v}$ and $\mathbf{Q}_{a}$ are optimised over $q_{v} \in$ $\{0.05,0.5,5,50\}$ and $q_{a} \in\{0.05,0.5,5,50\}$, respectively. For the model in (25) the true $\mathbf{R}$ and $\mathbf{Q}$ are used and the hyperparameters are optimised over $\theta \in\{25,50,75\}, \sigma^{2} \in\{1,10,50\}$ and $\delta \in\{50,75\}$. The state prior is the observation for the position and mean zero for the other states with covariance $\mathbf{P}_{0}=\mathbf{R}$ for the proposed model, $\mathbf{P}_{0}=\operatorname{diag}\left(\mathbf{R}, 25 \mathbf{I}_{2}\right)$ for the NCV model and $\mathbf{P}_{0}=\operatorname{diag}\left(\mathbf{R}, 25 \mathbf{I}_{4}\right)$ for the NCA model.

The root mean squared error (RMSE) of the position and velocity for each estimator and each configuration is presented in Tab. I, where the proposed estimator is denoted GP. It is clear that the proposed method gives better performance than the standard models in this scenario. However, the performance gain reduces with increasing observation frequency and increasing process noise. The averaged normalised estimation 
TABLE I

RMSE RESULTS OF SIMULATIONS.

\begin{tabular}{cccccccc}
\hline \multicolumn{2}{c}{ Config. } & \multicolumn{3}{c}{ Position RMSE $(\mathrm{m})$} & \multicolumn{3}{c}{ Velocity RMSE } \\
$\mathbf{S}$ & $\mathbf{q}$ & NCV & NCA & GP & NCV & NCA & GP \\
\hline 1 & 2 & 2.66 & 2.53 & 2.13 & 2.94 & 2.15 & 0.69 \\
1 & 20 & 2.99 & 3.01 & 2.97 & 26.26 & 36.78 & 2.36 \\
1 & 50 & 3.02 & 3.13 & 2.99 & 64.27 & 89.73 & 4.88 \\
2 & 2 & 4.24 & 3.99 & 2.86 & 2.83 & 2.55 & 0.84 \\
5 & 0 & 6.67 & 8.49 & 3.77 & 2.42 & 3.29 & 1.23 \\
5 & 0.5 & 6.74 & 8.59 & 3.87 & 2.43 & 3.32 & 1.23 \\
5 & 2 & 7.77 & 10.01 & 4.91 & 2.68 & 3.85 & 1.22 \\
5 & 5 & 11.98 & 15.74 & 8.29 & 3.79 & 6.00 & 1.42 \\
5 & 20 & 40.41 & 71.93 & 29.05 & 11.96 & 29.52 & 3.19 \\
5 & 50 & 101.46 & 177.33 & 71.29 & 30.03 & 72.66 & 5.83 \\
10 & 2 & 18.9 & 26.88 & 10.77 & 3.92 & 5.84 & 1.86 \\
\hline
\end{tabular}

TABLE II

ANEES RESULTS OF SIMULATIONS.

\begin{tabular}{ccccc}
\hline \multicolumn{3}{c}{ Config. } & \multicolumn{3}{c}{ ANEES } \\
\hline S & $\mathbf{q}$ & NCV & NCA & GP \\
\hline 1 & 2 & 0.93 & 1.05 & 1.00 \\
1 & 20 & 1.00 & 1.01 & 1.00 \\
1 & 50 & 1.02 & 1.09 & 1.00 \\
2 & 2 & 0.78 & 1.05 & 1.00 \\
5 & 0 & 0.33 & 0.67 & 1.16 \\
5 & 0.5 & 0.33 & 0.69 & 1.06 \\
5 & 2 & 0.37 & 0.89 & 1.03 \\
5 & 5 & 0.6 & 2.02 & 1.01 \\
5 & 20 & 4.72 & 1.51 & 1.01 \\
5 & 50 & 0.56 & 8.13 & 1.00 \\
10 & 2 & 0.22 & 0.43 & 1.30 \\
\hline
\end{tabular}

error squared (ANEES), see Tab. II, also shows that the proposed estimator is in general consistent, unlike the estimators using the standard models. Two notable exceptions in Tab. II are the configurations with no process noise and a long time between the observations. In the first case, the noise introduced by the GP will have a larger relative effect on the estimate, causing inconsistencies. In the second case the use of a noisy input to the GP starts having effect. The velocity computed for the proposed method is obtained as the predicted velocity at the estimated location of the target.

\section{B. Sea-Ice Tracking Application}

The proposed method is applied to a Terrestrial Radar Interferometer (TRI) [31] dataset collected to track drift ice by NORCE* $^{*}$ in Kongsfjorden on Svalbard. The dataset consists of radar samples taken every $3 \mathrm{~min}$ over $41 \mathrm{~h}$. An example of the data transformed into Cartesian coordinates is presented in Fig. 2.

Detections in the dataset are obtained as described in [32]. The purpose of the example is to evaluate the model, hence data association is not considered in the evaluation. More investigation is required into how to incorporate the method into a tracker in order to achieve reasonable computational complexity. A global nearest neighbour (GNN) tracker [33] is applied on the full dataset using a NCV model [30]. Detections associated to tracks of at least $30 \mathrm{~min}$ are stored. For each track $30 \%$ of the detections are kept to simulate a lower sampling rate.

*NORCE Norwegian Research Centre

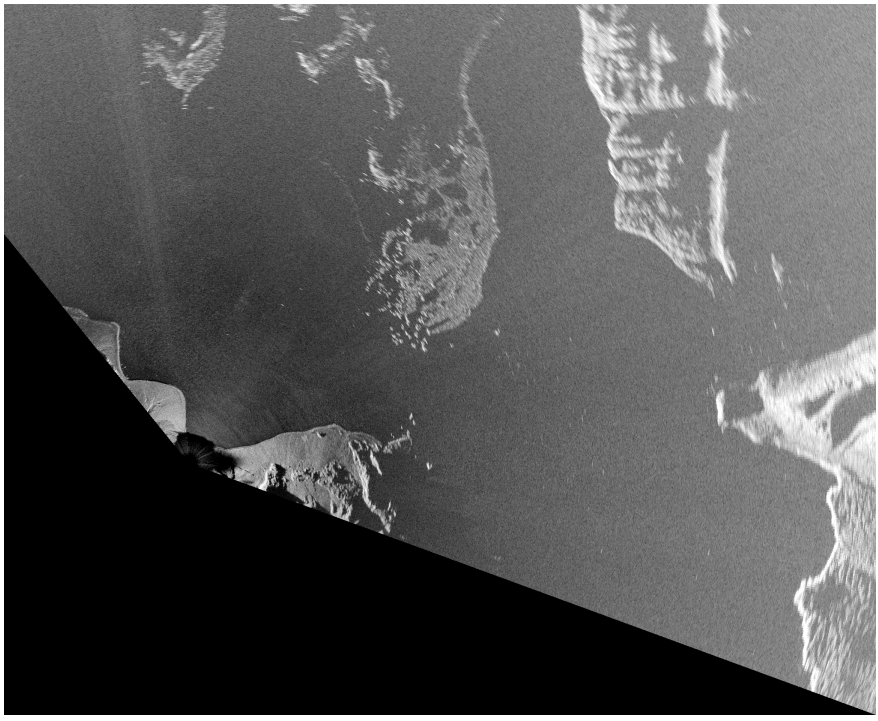

Fig. 2. Radar sample from the TRI dataset. The backscattered intensity is transformed to a local Cartesian coordinate system.

The icebergs are assumed to have independent velocities, but are affected by a common acceleration from currents and winds, which are subject to change over time. The model for the icebergs used for estimation is therefore chosen as

$$
\begin{aligned}
\mathbf{x}_{k_{1}^{i}}^{i} & \sim \mathcal{N}\left(\overline{\mathbf{x}}_{0}^{i}, \mathbf{P}_{0}\right), \\
\mathbf{x}_{k}^{i} & =\mathbf{A} \mathbf{x}_{k-1}^{i}+\mathbf{B}\left(\tilde{\mathbf{K}}_{\cdot u}\left(\mathbf{D} \mathbf{x}_{k-1}^{i}\right) \mathcal{W}_{k}+\right. \\
& \left.\quad \mathbf{v}_{k}^{i f}\left(\mathbf{D} \mathbf{x}_{k-1}^{i}\right)\right)+\mathbf{v}_{k}^{i}, \\
\mathbf{y}_{j}^{i} & =\mathbf{C} \mathbf{x}_{k_{j}^{i}}^{i}+\mathbf{e}_{j}^{i}, \\
\mathcal{W}_{0} & \sim \mathcal{N}\left(\mathbf{0}, \tilde{\mathbf{K}}_{u u}^{-1}\right), \\
\mathcal{W}_{k} & =e^{-\alpha T_{s}} \mathcal{W}_{k-1}+\mathbf{v}_{k}^{w},
\end{aligned}
$$

where the state $\mathbf{x}_{k}^{i}$ is the position $[\mathrm{m}]$ and the velocity $[\mathrm{m} / \mathrm{h}]$ of iceberg $i=1, \ldots, I$ at time step $k \in\left\{k_{1}^{i}, \ldots, k_{J}^{i}\right\}$ and $k_{j}^{i}$ denotes the time step of observation $j=1, \ldots, J$ for the target. The kernel is chosen as in (8) and $\mathbf{v}_{k}^{w} \sim \mathcal{N}(\mathbf{0},(1-$ $\left.\left.e^{-2 \alpha T_{s}}\right) \tilde{\mathbf{K}}_{u u}^{-1}\right)$ with $T_{s}=0.05 \mathrm{~h}$. The system matrix is $\mathbf{A}=$ $\left(\begin{array}{cc}1 & T_{s} \\ 0 & 1\end{array}\right) \otimes \mathbf{I}_{2}, \mathbf{B}=\left(\begin{array}{c}T_{s}^{2} / 2 \\ T s\end{array}\right) \otimes \mathbf{I}_{2}, \mathbf{v}_{k}^{i} \sim \mathcal{N}\left(\mathbf{0}, \mathbf{Q}_{v}\right)$ with $\mathbf{Q}_{v}$ defined as in (26a) and the matrix $\mathbf{D}$ selects the position states. The observation noise is given by $\mathbf{e}_{j}^{i} \sim \mathcal{N}\left(\mathbf{0}, r^{2} \mathbf{I}_{2}\right)$ and the observation matrix $\mathbf{C}$ selects the position states. The state prior is the first observation for the position and zero velocity with $\mathbf{P}_{0}=\operatorname{diag}\left(r^{2} \mathbf{I}_{2}, 500^{2} \mathbf{I}_{2}\right)$. For comparison, the $\mathrm{NCV}$ and $\mathrm{NCA}$ models are considered with the respective covariance matrices $\mathbf{Q}_{v}$ and $\mathbf{Q}_{a}$ as defined in (26).

The hyperparameters are optimised for the NCV model over $q \in\{42.4,141.4,424.3\}$, for the NCA model over $q \in\{84.9, \underline{282.8}, 848.5\}$ and over $r \in\{5, \underline{15}, 50\} \mathrm{m}$ for both models with regards to maximum likelihood, with the optimal parameters underlined.

For the proposed model the hyperparameters are optimised over $q \in\{42.4, \underline{141.4}, 424.3\}, \theta=\delta \in\{500, \underline{750}, 1000\} \mathrm{m}$, $\sigma \in\{63.2, \underline{200}, 632.5\} \mathrm{m} / \mathrm{h}^{2}$ and $\alpha \in\{0.01, \underline{0.05}\}$, with the optimal parameters underlined. Since the true iceberg locations 
TABLE III

PREDICTION ERRORS FOR THE SEA-ICE TRACKING APPLICATION.

\begin{tabular}{lccc}
\hline Method & NCV & NCA & GP \\
\hline Quadratic Norm & 6.13 & 7.27 & 6.00 \\
Maximum Likelihood & 385877 & 403723 & 383336 \\
\hline
\end{tabular}

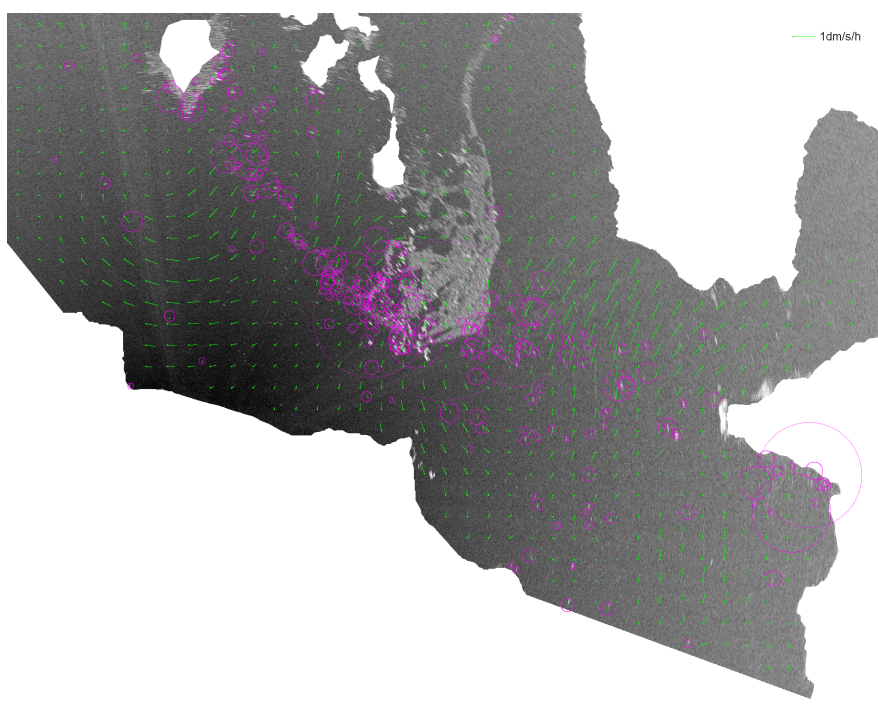

Fig. 3. The tracks and current acceleration estimation of the sea-ice tracking application.

are not available, the prediction error method (PEM) [34] is used to compare the models. The method is applied both with a quadratic norm for the prediction errors and in the maximum likelihood form. The results are presented in Tab. III, showing the negative log-likelihoods for the latter. The proposed method performs slightly better in this setup compared to the traditional models. The tracks and the estimated acceleration function for a single instant of time is presented in Fig. 3.

Using the various methods for implementation considered in Section IV, the computation time reduces significantly and it is feasible to run an optimisation algorithm. The particular approximations used are zeroing of cross-correlation blocks with an absolute sum less than $1 \cdot 10^{-4}$ and gating of targets and inducing inputs within a distance of $2.1 \theta$ and $5.7 \theta$, respectively. The log-likelihood is optimised over the parameters $q$ and $r$ for the NCV model, resulting in $q=161.3$ and $r=12.4 \mathrm{~m}$. The same parameters are used for the GP model, which is then optimised over $\theta, \delta=2 \theta / 3$ and $\sigma$, resulting in $\theta=739 \mathrm{~m}$ and $\sigma=3.5 \mathrm{~m} / \mathrm{h}^{2}$. The negative log-likelihood is 382359 and 380522 for the NCV and GP models, respectively, showing slightly better performance for the proposed model.

\section{CONCLUSION}

External influences on target dynamics, such as winds, paths or obstacles, are often modelled as noise. They have instead been incorporated explicitly as unknown functions of the state modelled as Gaussian processes. By approximating the functions as sparse Gaussian processes using inducing variables the influences can be learned online together with the inference of the states using an extended Kalman filter.
By a simple extension, the function can also be allowed to change over time. Several approaches to achieve a faster implementation are discussed.

The main advantages of the proposed method are that recursive inference and learning for dynamical systems with unknown influences are integrated into a single framework, that learning can be limited to a particular influence or part of the system, that the complexity does not increase with larger datasets and that time-varying influences can be considered.

The method is applied in a simulated scenario with multiple targets. The target velocities are given as a static function of their position. The method is shown to outperform traditional motion models in this scenario and to estimate the velocity function accurately where data is available. The method is also applied in a sea-ice tracking application where the acceleration of the icebergs is modelled by a function that changes over time.

Although the initial results for the proposed model are promising, there are several opportunities for improvement, both theoretically and practically. A few of them are here discussed briefly.

A practical problem is that the region of operation needs to be determined beforehand to select the inducing inputs. Modifying the inputs online was shown in Sec. IV-B to be non-trivial, so a different approach is necessary.

The hyperparameters are selected manually at design time using maximum likelihood estimation or expert knowledge. A more systematic approach to determine good hyperparmeters would improve the usability of the model.

A theoretic analysis of the impact of the proposed implementation approximations is required to safely decouple dynamic systems and inducing variables when the dependence is small.

A method to tractably incorporate the model into a data association framework, possibly considering multiple association hypotheses, is essential for use in multi-target tracking.

Additionally, the performance improvement that could potentially be achieved by the method should be assessed.

\section{ACKNOWLEDGEMENT}

This work has been supported by funding from the Vinnova Industry Excellence Center LINK-SIC. Discussions with Martin Skoglund, Oticon, have been very appreciated.

\section{APPENDIX A}

GAUSSIAN MANIPULATION

Proposition 1. The following relationship between the probability distributions hold,

$$
\begin{aligned}
& \mathcal{N}(\mathbf{y} ; \mathbf{H x}, \mathbf{R}) \\
& \quad \propto \mathcal{N}\left(\mathbf{x} ;\left(\mathbf{H}^{T} \mathbf{R}^{-1} \mathbf{H}\right)^{-1} \mathbf{H}^{T} \mathbf{R}^{-1} \mathbf{y},\left(\mathbf{H}^{T} \mathbf{R}^{-1} \mathbf{H}\right)^{-1}\right),
\end{aligned}
$$

assuming full rank of $\mathbf{R}$ and $\mathbf{H}^{T} \mathbf{R}^{-1} \mathbf{H}$, where $\propto$ defines equality up to a constant independent of $\mathbf{x}$. 
Proof. Manipulations of the density function shows

$$
\begin{aligned}
& \mathcal{N}(\mathbf{y} ; \mathbf{H x}, \mathbf{R}) \\
& \propto \exp \left(-\frac{1}{2}(\mathbf{y}-\mathbf{H} \mathbf{x})^{T} \mathbf{R}^{-1}(\mathbf{y}-\mathbf{H} \mathbf{x})\right) \\
& \propto \exp \left(-\frac{1}{2}\left(\mathbf{x}^{T} \mathbf{H}^{T} \mathbf{R}^{-1} \mathbf{H} \mathbf{x}-2 \mathbf{y}^{T} \mathbf{R}^{-1} \mathbf{H} \mathbf{x}\right)\right) \\
&= \exp \left(-\frac{1}{2}\left(\mathbf{x}^{T} \mathbf{H}^{T} \mathbf{R}^{-1} \mathbf{H} \mathbf{x}\right.\right. \\
&\left.\left.\quad-2 \mathbf{y}^{T} \mathbf{R}^{-1} \mathbf{H}\left(\mathbf{H}^{T} \mathbf{R}^{-1} \mathbf{H}\right)^{-1} \mathbf{H}^{T} \mathbf{R}^{-1} \mathbf{H} \mathbf{x}\right)\right) \\
& \propto \exp \left(-\frac{1}{2}\left(\mathbf{x}-\left(\mathbf{H}^{T} \mathbf{R}^{-1} \mathbf{H}\right)^{-1} \mathbf{H}^{T} \mathbf{R}^{-1} \mathbf{y}\right)^{T}\right. \\
&\left.\left.\quad \mathbf{H}^{T} \mathbf{R}^{-1} \mathbf{H}\left(\mathbf{x}-\left(\mathbf{H}^{T} \mathbf{R}^{-1} \mathbf{H}\right)^{-1} \mathbf{H}^{T} \mathbf{R}^{-1} \mathbf{y}\right)\right)\right) \\
& \propto \mathcal{N}\left(\mathbf{x} ;\left(\mathbf{H}^{T} \mathbf{R}^{-1} \mathbf{H}\right)^{-1} \mathbf{H}^{T} \mathbf{R}^{-1} \mathbf{y},\left(\mathbf{H}^{T} \mathbf{R}^{-1} \mathbf{H}\right)^{-1}\right)
\end{aligned}
$$

\section{APPENDIX B \\ JACOBIAN OF GAUSSIAN NOISE}

Proposition 2. Given the stochastic variable

$$
\mathbf{v} \sim \mathcal{N}(\boldsymbol{\mu}(\mathbf{z}), \lambda(\mathbf{z}) \mathbf{I}),
$$

the Jacobian with regards to $\mathrm{z}$ is given by

$$
\begin{aligned}
\frac{\partial \mathbf{v}}{\partial \mathbf{z}} & =\frac{\partial \boldsymbol{\mu}}{\partial \mathbf{z}}+(\mathbf{v}-\boldsymbol{\mu}(\mathbf{z})) \frac{1}{2 \lambda(\mathbf{z})} \frac{\partial \lambda(\mathbf{z})}{\partial \mathbf{z}} \\
& =\frac{\partial \boldsymbol{\mu}}{\partial \mathbf{z}}+\frac{1}{2}(\mathbf{v}-\boldsymbol{\mu}(\mathbf{z})) \frac{\partial}{\partial \mathbf{z}}(\ln (\lambda(\mathbf{z}))
\end{aligned}
$$

Proof. Rewrite the stochastic variable as

$$
\mathbf{v}=\boldsymbol{\mu}(\mathbf{z})+\sqrt{\lambda(\mathbf{z})} \cdot \mathbf{n},
$$

where $\mathbf{n} \sim \mathcal{N}(\mathbf{0}, \mathbf{I})$, then

$$
\begin{aligned}
\frac{\partial \mathbf{v}}{\partial \mathbf{z}} & =\frac{\partial \boldsymbol{\mu}}{\partial \mathbf{z}}+\mathbf{n} \frac{1}{2 \sqrt{\lambda(\mathbf{z})}} \frac{\partial \lambda(\mathbf{z})}{\partial \mathbf{z}} \\
& =\frac{\partial \boldsymbol{\mu}}{\partial \mathbf{z}}+(\mathbf{v}-\boldsymbol{\mu}(\mathbf{z})) \frac{1}{2 \lambda(\mathbf{z})} \frac{\partial \lambda(\mathbf{z})}{\partial \mathbf{z}} \\
& =\frac{\partial \boldsymbol{\mu}}{\partial \mathbf{z}}+\frac{1}{2}(\mathbf{v}-\boldsymbol{\mu}(\mathbf{z})) \frac{\partial}{\partial \mathbf{z}}(\ln (\lambda(\mathbf{z}))
\end{aligned}
$$

\section{APPENDIX C}

MARgINALISATION OF INDUCING VARIABLES

The conditional distribution of $\mathcal{F}$ given $\mathcal{W}$ is

$$
\mathcal{F} \mid \mathcal{W} \sim \mathcal{N}\left(\tilde{\mathbf{K}}_{f u} \mathcal{W}, \tilde{\boldsymbol{\Lambda}}\right)
$$

The joint distribution is then given by

$$
\begin{gathered}
p(\mathcal{Y}, \mathcal{X}, \mathcal{F}, \mathcal{W})=p(\mathcal{Y} \mid \mathcal{X}) p(\mathcal{X} \mid \mathcal{F}) p(\mathcal{F} \mid \mathcal{W}) p(\mathcal{W}) \\
=\prod_{k=1}^{K} \mathcal{N}\left(\mathbf{y}_{k} ; \mathbf{C}_{k} \mathbf{x}_{k}, \mathbf{R}_{k}\right) \cdot \mathcal{N}\left(\mathbf{x}_{0} ; \overline{\mathbf{x}}_{0}, \mathbf{P}_{0}\right) \\
\prod_{k=1}^{K} \mathcal{N}\left(\mathbf{x}_{k} ; \mathbf{A}_{k} \mathbf{x}_{k-1}+\mathbf{B}_{k} \mathbf{f}_{k-1}, \mathbf{Q}_{k}\right) . \\
\mathcal{N}\left(\mathcal{F} ; \tilde{\mathbf{K}}_{f u} \mathcal{W}, \tilde{\boldsymbol{\Lambda}}\right) \cdot \mathcal{N}\left(\mathcal{W} ; \mathbf{0}, \tilde{\mathbf{K}}_{u u}^{-1}\right)
\end{gathered}
$$

Integration over $\mathcal{F}$ gives

$$
\begin{aligned}
& \int_{\mathcal{F}} \prod_{k=1}^{K} \mathcal{N}\left(\mathbf{x}_{k} ; \mathbf{A}_{k} \mathbf{x}_{k-1}+\mathbf{B}_{k} \mathbf{f}_{k-1}, \mathbf{Q}_{k}\right) \\
& \mathcal{N}\left(\mathcal{F} ; \tilde{\mathbf{K}}_{f u} \mathcal{W}, \tilde{\mathbf{\Lambda}}\right) d \mathcal{F} \\
& =\prod_{k=1}^{K} \int_{\mathbf{f}_{k-1}} \mathcal{N}\left(\mathbf{x}_{k} ; \mathbf{A}_{k} \mathbf{x}_{k-1}+\mathbf{B}_{k} \mathbf{f}_{k-1}, \mathbf{Q}_{k}\right) \\
& \quad \mathcal{N}\left(\mathbf{f}_{k-1} ; \tilde{\mathbf{K}}_{f u}^{k-1} \mathcal{W}, \tilde{\mathbf{\Lambda}}_{k-1}\right) d \mathbf{f}_{k-1} \\
& =\prod_{k=1}^{K} \mathcal{N}\left(\mathbf{x}_{k} ; \mathbf{A}_{k} \mathbf{x}_{k}+\mathbf{B}_{k} \tilde{\mathbf{K}}_{f u}^{k-1} \mathcal{W}, \mathbf{B}_{k} \tilde{\mathbf{\Lambda}}_{k-1} \mathbf{B}_{k}^{T}+\mathbf{Q}_{k}\right)
\end{aligned}
$$

which results in the marginalized joint distribution

$$
\begin{aligned}
& p(\mathcal{Y}, \mathcal{X}, \mathcal{W})=\int_{\mathcal{F}} p(\mathcal{Y}, \mathcal{X}, \mathcal{F}, \mathcal{W}) d \mathcal{F} \\
&=\prod_{k=1}^{K} \mathcal{N}\left(\mathbf{y}_{k} ; \mathbf{C}_{k} \mathbf{x}_{k}, \mathbf{R}_{k}\right) \cdot \mathcal{N}\left(\mathbf{x}_{0} ; \overline{\mathbf{x}}_{0}, \mathbf{P}_{0}\right) . \\
& \prod_{k=1}^{K} \mathcal{N}\left(\mathbf{x}_{k} ; \mathbf{A}_{k} \mathbf{x}_{k-1}+\mathbf{B}_{k} \tilde{\mathbf{K}}_{f u}^{k-1} \mathcal{W}, \mathbf{B}_{k} \tilde{\mathbf{\Lambda}}_{k-1} \mathbf{B}_{k}^{T}+\mathbf{Q}_{k}\right) . \\
& \mathcal{N}\left(\mathcal{W} ; \mathbf{0}, \tilde{\mathbf{K}}_{u u}^{-1}\right) .
\end{aligned}
$$

\section{REFERENCES}

[1] H. A. P. Blom, "An efficient filter for abruptly changing systems," in Proceedings of the 23rd IEEE Conference on Decision and Control, Las Vegas, NV, USA, Dec. 1984, pp. 656-658.

[2] S. Coraluppi, C. Carthel, P. Braca, and L. Millefiori, "The mixed Ornstein-Uhlenbeck process and context exploitation in multi-target tracking," in Proceedings of the 19th International Conference on Information Fusion, Heidelberg, Germany, Jul. 2016, pp. 217-224.

[3] L. M. Millefiori, P. Braca, K. Bryan, and P. Willett, "Modeling vessel kinematics using a stochastic mean-reverting process for long-term prediction," IEEE Transactions on Aerospace and Electronic Systems, vol. 52, no. 5, pp. 2313-2330, Oct. 2016.

[4] G. Pallotta, S. Horn, P. Braca, and K. Bryan, "Context-enhanced vessel prediction based on Ornstein-Uhlenbeck processes using historical AIS traffic patterns: Real-world experimental results," in Proceedings of the 17th International Conference on Information Fusion, Salamanca, Spain, Jul. 2014.

[5] S. Hexeberg, A. L. Flåten, B. O. H. Eriksen, and E. F. Brekke, "AIS-based vessel trajectory prediction," in Proceedings of the 20th International Conference on Information Fusion, Xi'an, China, Jul. 2017.

[6] G. Vivone, P. Braca, and J. Horstmann, "Knowledge-based multitarget ship tracking for hf surface wave radar systems," IEEE Transactions on Geoscience and Remote Sensing, vol. 53, no. 7, pp. 3931-3949, Jul. 2015.

[7] F. Gustafsson, F. Gunnarsson, N. Bergman, U. Forssell, J. Jansson, R. Karlsson, and P. J. Nordlund, "Particle filters for positioning, navigation, and tracking," IEEE Transactions on Signal Processing, vol. 50, no. 2, pp. 425-437, Feb. 2002.

[8] M. Ulmke and W. Koch, "Road-map assisted ground moving target tracking," IEEE Transactions on Aerospace and Electronic Systems, vol. 42, no. 4, pp. 1264-1274, Oct. 2006.

[9] D. Ellis, E. Sommerlade, and I. Reid, "Modelling pedestrian trajectory patterns with Gaussian processes," in Proceedings of the 12th International Conference on Computer Vision Workshops, Kyoto, Japan, Sep. 2009, pp. 1229-1234.

[10] K. Kim, D. Lee, and I. Essa, "Gaussian process regression flow for analysis of motion trajectories," in Proceedings of the 13th International Conference on Computer Vision Workshops, Barcelona, Spain, Nov. 2011, pp. 1164-1171.

[11] Q. Tran and J. Firl, "Modelling of traffic situations at urban intersections with probabilistic non-parametric regression," in Proceedings of the IEEE Intelligent Vehicles Symposium, Gold Coast, Australia, Jun. 2013, pp. 334-339. 
[12] M. Tiger and F. Heintz, "Online sparse Gaussian process regression for trajectory modeling," in Proceedings of the 18th International Conference on Information Fusion, Washington D.C., USA, Jul. 2015, pp. 782-791.

[13] C. Rasmussen and C. Williams, Gaussian Processes for Machine Learning, ser. Adaptive Computation and Machine Learning. Cambridge, MA, USA: MIT Press, Jan. 2006.

[14] J. Quiñonero Candela and C. E. Rasmussen, "A unifying view of sparse approximate Gaussian process regression," Journal of Machine Learning Research, vol. 6, pp. 1939-1959, Dec. 2005.

[15] G. L. Smith, S. F. Schmidt, and L. A. McGee, Application of Statistical Filter Theory to the Optimal Estimation of Position and Velocity on Board a Circumlunar Vehicle. Washington D. C., USA: National Aeronautics and Space Administration, 1962.

[16] M. P. Deisenroth, M. F. Huber, and U. D. Hanebeck, "Analytic momentbased Gaussian process filtering," in Proceedings of the 26th Annual International Conference on Machine Learning, New York, NY, USA, Jun. 2009, pp. 225-232.

[17] R. Turner, M. Deisenroth, and C. Rasmussen, "State-space inference and learning with Gaussian processes," in Proceedings of the 13th International Conference on Artificial Intelligence and Statistics, vol. 9, Sardinia, Italy, May 2010, pp. 868-875.

[18] R. Frigola, F. Lindsten, T. B. Schön, and C. E. Rasmussen, "Bayesian inference and learning in Gaussian process state-space models with particle MCMC," in Proceedings of the 26th International Conference on Neural Information Processing Systems - Volume 2, Lake Tahoe, NV, USA, 2013, pp. 3156-3164.

[19] A. Solin and S. Särkkä, "Hilbert space methods for reduced-rank Gaussian process regression," Statistics and Computing, 2019.

[20] M. F. Huber, "Recursive Gaussian process: On-line regression and learning," Pattern Recognition Letters, vol. 45, pp. 85-91, 2014.

[21] H. Bijl, J.-W. van Wingerden, T. B. Schön, and M. Verhaegen, "Online sparse Gaussian process regression using FITC and PITC approximations," IFAC-PapersOnLine, vol. 48, no. 28, pp. 703-708, 2015.

[22] H. Bijl, T. B. Schön, J. van Wingerden, and M. Verhaegen, "System identification through online sparse Gaussian process regression with input noise," IFAC Journal of Systems and Control, vol. 2, pp. 1-11, Dec. 2017.

[23] N. Wahlström and E. Özkan, "Extended target tracking using Gaussian processes," IEEE Transactions on Signal Processing, vol. 63, no. 16, pp. 4165-4178, Aug. 2015.

[24] M. Kok and A. Solin, "Scalable magnetic field SLAM in 3D using Gaussian process maps," in Proceedings of the 21st International Conference on Information Fusion, Cambridge, UK, Jul. 2018, pp. 1353-1360.

[25] S. Särkkä, Bayesian Filtering and Smoothing. Cambridge, United Kingdom: Cambridge University Press, 2013.

[26] A. Solin, M. Kok, N. Wahlström, T. B. Schön, and S. Särkkä, "Modeling and interpolation of the ambient magnetic field by Gaussian processes," IEEE Transactions on Robotics, vol. 34, no. 4, pp. 1112-1127, Aug. 2018.

[27] A. McHutchon and C. E. Rasmussen, "Gaussian process training with input noise," in Proceedings of the 24th International Conference on Neural Information Processing Systems, Granada, Spain, Dec. 2011, pp. 1341-1349.

[28] R. E. Kalman, "A new approach to linear filtering and prediction problems," Transactions of the ASME-Journal of Basic Engineering, vol. 82, no. Series D, pp. 35-45, 1960.

[29] A. J. Storkey, "Truncated covariance matrices and toeplitz methods in Gaussian processes," in Proceedings of the Ninth International Conference on Artificial Neural Networks, vol. 1, Edinburgh, United Kingdom, Sep. 1999, pp. 55-60 vol.1.

[30] R. X. Li and V. P. Jilkov, "Survey of maneuvering target tracking. Part I: Dynamic models," IEEE Transactions on Aerospace and Electronic Systems, vol. 39, no. 4, pp. 1333-1364, Oct. 2003.

[31] D. Voytenko, T. H. Dixon, M. E. Luther, C. Lembke, I. M. Howat, and S. de la Peña, "Observations of inertial currents in a lagoon in southeastern Iceland using terrestrial radar interferometry and automated iceberg tracking," Computers \& Geosciences, vol. 82, pp. 23-30, 2015.

[32] J. Olofsson, C. Veibäck, and G. Hendeby, "Sea ice tracking with a spatially indexed labeled multi-Bernoulli filter," in Proceedings of the 20th International Conference on Information Fusion, Xi'an, China, Jul. 2017, pp. 376-383.

[33] S. S. Blackman and R. Popoli, Design and Analysis of Modern Tracking Systems. Artech House, 1999

[34] L. Ljung, System Identification: Theory for the User, 2nd ed. Upper Saddle River, NJ, USA: Prentice Hall, 1999.
Clas Veibäck is a post-doctoral researcher in the Division of Automatic Control, Department of Electrical Engineering, Linköping University, Sweden. $\mathrm{He}$ received his Ph.D. in Automatic Control from Linköping University in December 2018 and his M.Sc. in engineering physics from Uppsala University, Sweden in December 2011.

His research projects have mainly involved tracking using cameras, microphones and radars as sensors, both stationary and as part of moving platforms. He is interested in sensor fusion and signal processing in general, with a focus on target tracking.

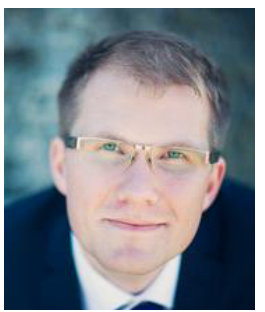

Jonatan Olofsson is a post-doctoral researcher in the Division of Automatic Control, Department of Electrical Engineering, Linköping University, Sweden. Research interests include the field of sensor fusion, and its practical applications to real-world data. He received his M.Sc. degree in Applied Physics and Electrical Engineering from Linköping University, Sweden, in 2012 and 2019 his Ph.D. degree in the field of Cybernetics at the Norwegian University of Science and Technology (NTNU) through MarineUAS, a Marie Skłodowska-Curie ITN performing research on the use of Unmanned Aerial Systems on the European coast.

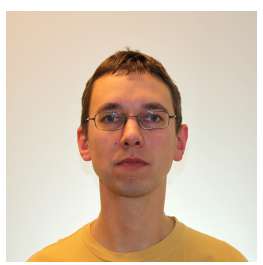

Tom Rune Lauknes (M '01) is a Senior Researcher with NORCE Norwegian Research Centre, Troms $\emptyset$, Norway, since 2005. He received the Cand.Sci. (M.Sc.) degree (2002) and the Ph.D. (2011) in physics, both from the University of Troms $\varnothing$, Troms $\varnothing$. He was a Visiting Researcher with the Radar Interferometry Group at Stanford University (2007-2008), a Visiting Research Fellow at Centre for Space Research, University of Texas at Austin (2010), and a Visiting Scholar at Depart-

Barbara (2014). ment of Geography, University of California, Santa

He has long experience with the use of radar interferometric methods for geophysical applications. He is involved in cryospheric and polar science research projects related to monitoring of Arctic sea ice and calving glaciers, using a combination of terrestrial and satellite radar remote sensing methods and Unmanned Aerial Vehicle (UAV). He is a member of the American Geophysical Union.

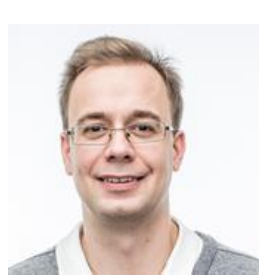

Gustaf Hendeby (SM '17) is an Associate Professor in the Division of Automatic Control at Linköping University, Sweden. He received his M.Sc. in Applied Physics and Electrical Engineering in 2002 and his Ph.D. in Automatic Control in 2008, both from Linköping University. He worked as a Senior Researcher at the German Research Centre for Artificial Intelligence (DFKI) 2009-2011, and Senior Scientist at the Swedish Defence Research Agency (FOI) and held an adjunct Associate Professor position at Linköping University 2011-2015.

His main research interests are stochastic signal processing and sensor fusion with applications to nonlinear problems, target tracking, and simultaneous localization and mapping (SLAM). He has experience of both theoretical analysis as well as implementation aspects. 